\title{
Neuromodulation for chronic urologic pain
}

\author{
Dean Elterman, MD, MSc, FRCSC
}

Division of Urology, Toronto Western Hospital, University of Toronto, Toronto ON

Cite as: Can Urol Assoc J 2018;12(6Supp|3):S184-5. http://dx.doi.org/10.5489/cuai.5332

\section{Introduction}

Chronic urologic pain, be it bladder pain syndrome, prostatitis, or some other form of urologic chronic pelvic pain syndrome (UCPPS), may present in a variety of symptoms and severities. A multimodal approach, as outlined in this supplement, is generally required to manage these difficultto-treat conditions. One option available may be to electrically alter the afferent pain signals within the pelvis through sacral neuromodulation (SNM). The bladder and pelvic floor are innervated via sacral nerve roots, which transmit afferent sensory signals up to the brain. The S3 sacral nerve root is most specifically associated with sensory function of the detrusor and pelvic floor muscles. In addition to afferent sensory and efferent motor nerves responsible for bladder and bowel function, pain signals are transmitted through $\mathrm{C}$-fibres and A $\delta$-fibers that travel along the same path.

SNM provides low-amplitude electrical stimulation to the S3 nerve root, activating inhibitory signals and modulating afferent sensory messages. SNM is Health Canada-approved for urinary frequency-urgency, urgency urinary incontinence, chronic non-obstructive urinary retention, and fecal incontinence. The treatment of UCPPS, while supported by the literature, is technically an off-label application of SNM. However, UCPPS is frequently (for interstitial cystitis/bladder pain syndrome [IC/BPS] almost always) associated with significant and bothersome storage symptoms. The process to ultimately implant an SNM system, including an implantable pulse generator (IPG; "battery" or "pacemaker") connected to a lead with electrodes, requires two steps. The first step is a screening trial, which provides temporary and reversible stimulation over a short duration (4-14 days) to assess for efficacy. The desired outcome to move forward with formal implantation of an SNM system is a $50 \%$ improvement in symptoms (i.e., $50 \%$ reduction in pain; $50 \%$ reduction in analgesic use or improvement in storage symptoms if they are a dominant impact on quality of life). The screening trial may be performed in an office setting with a percutaneous nerve evaluation (PNE), whereby a monopolar, untined lead is implanted under local anesthesia and is connected to the temporary external neurostimulator (ENS). After 4-7 days, everything is removed and the decision to proceed with a full SNM system implantation is made based on a minimum $50 \%$ improvement in symptoms. Alternatively, a "staged" implant can be performed in the operating room, whereby the permanent ("chronic") quadriploar, tined lead is placed fluoroscopically and is connected to the same temporary ENS for the duration of the short evaluative trial. After around two weeks, if the patient achieves a 50\% improvement, the already implanted lead is internalized and connected to a surgically implanted IPG (placed subcutaneously in the upper buttock). See Fig. 1 for a summary of the steps of SNM.

The reported success rates for treating UCPPS are $51-77 \%$ during screening trials and $40-72 \%$ following full SNM implantation (followup range 5-87 months).

Percutaneous tibial nerve stimulation (PTNS) is an alternative modality of neuromodulation altering pelvic function. The tibial nerve shares a common origin with the sacral nerve innervating the pelvic floor and bladder. Thus, stimulating this peripheral nerve can result in alterations of pelvic pain, along with bladder and bowel function. PTNS uses a $34 \mathrm{G}$ needle inserted $3-5 \mathrm{~cm}$ above the medial malleolus, connected to an external electrical stimulation device. Patients attend 10-12 weekly treatment sessions lasting 30 minutes, followed by monthly sessions to maintain the efficacy of the PTNS. Direct pudendal neuromodulation has been described to treat some UCPPS, however, only a handful of sites offer the therapy, none of which are in Canada.

\section{Practice tips}

\section{Tip 1: Remember that SNM may be an option}

There is a long list of familiar and conventional therapies we routinely use to treat UCPPS. SNM is generally a lesser known therapy, with few centres offering the treatment across Canada. By understanding the basic premise of the therapy and how pain is largely a neurological phenomenon, hopefully SNM will move up your list of therapeutic options. 


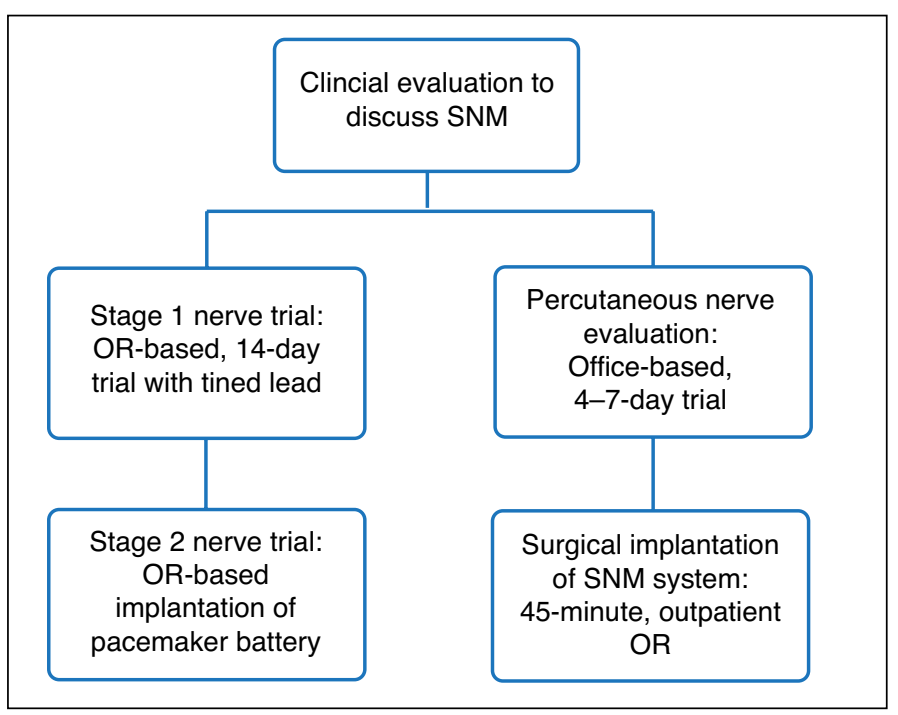

Fig. 1. Algorithm - Steps of sacral neuromodulation (SNM) for urologic chronic pelvic pain syndrome (UCPPS). OR: operating room.

\section{Tip 2: Know the centres that provide SNM near you}

There are several dedicated academic centres that provide SNM therapy to the population of that province or adjacent provinces. SNM is currently offered in Alberta, Ontario, Quebec, and Nova Scotia.

\section{Tip 3: Don't wait for SNM to be the last option available}

As with bladder and bowel dysfunction, the more chronic and refractory the condition, the more challenging it is to treat it successfully. SNM does not need to be considered the therapy of last resort in patients with UCPPS. SNM should be part of the multimodal approach to comprehensive pain management that is individualized for each patient.

\section{Tip 4: Every patient gets a screening trial first}

Many physicians and patients are apprehensive to embark on a permanent implantable "pacemaker." The advantage of SNM over other interventions is that the screening trial provides a non-invasive, fully reversible opportunity to assess efficacy prior to any surgery. Patients can choose if they wish to move forward with implantation.

Tip 5: Success rates are good, but set appropriate expectations, as improvements may dwindle over time in some patients

The reported success rates for treating UCPPS are $51-77 \%$ during screening trials. Following full SNM implantation, the mean reduction in pain scores was $40-72 \%$ (followup range 5-87 months). As with other therapies targeting UCPPS, setting expectations about what is a reasonable improvement and not necessarily a cure is important. My experience is the majority of UCPPS patients who receive SNM do get notable improvements, especially early on; however, they tend to habituate to their new reduced level of pain, focusing on that pain while forgetting how bad their previous pain was prior to SNM therapy.

Tip 6: Many patients have both urinary symptoms (frequency-urgency) and pelvic pain, thus a referral for SNM may benefit both conditions

Comorbid bothersome storage lower urinary tract symptoms can exacerbate UCPPS symptoms. For example, more frequent voids result in more instances of painful micturition. A referral to a centre for SNM with the primary intent of reducing storage symptoms may have a secondary benefit of reducing pain symptoms. Even if you think their pain is beyond control, consider SNM for their frequency-urgency. There's a good chance benefits will be seen on both symptom groups.

\section{Summary}

SNM provides gentle inhibitory stimulation of the pelvic floor and organs via the S3 nerve root, resulting in notable reductions in UCPPS pain scores. This effective therapy should be used in conjunction with other approaches in the management of UCPPS. Practitioners should identify candidate patients early and refer to their regional centre performing SNM.

Competing interests: Dr. Elterman has attended advisory boards for, is a speaker for, and has received grant funding from Allergan, Astellas, Boston Scientific, Ferring, Medtronic, and Pfizer; and has participated in clinical trials supported by Astellas and Medtronic.

This paper has been peer reviewed.

\section{Recommended reading}

1. Marcelissen $T$, Jacobs $R$, van Kerrebroeck $P$, et al. Sacral neuromodulation as a treatment for chronic pelvic pain. J Urol 2011;186:387-93. https://doi.org/10.1016/i.juro.2011.02.2694

2. Tutolo $M$, Ammirati E, Heesakkers J, et al. Efficacy and safety of sacral and percutaneous tibial neuromodulation in non-neurogenic lower urinary tract dysfunction and chronic pelvic pain: A systematic review of the literature. Eur Urol 2018;73:406-18. htrps://doi.org/10.1016/j.eururo.2017.11.002

3. Wang J, Chen Y, Chen J, et al. Sacral neuromodulation for refractory bladder pain syndrome/interstitial cystitis: A global systematic review and meta-analysis. Sci Rep 2017;7: 11031. https://doi. org/10.1038/s41598-017-11062-x

Correspondence: Dr. Dean Elterman, Division of Urology, Toronto Western Hospital, University of Toronto, Toronto 0N; deanelterman@gmail.com 